\section{Systematic Space-Time Trellis Code Construction for Correlated Rayleigh-Fading Channels}

\author{
Zoltan Safar, Member, IEEE, and K. J. Ray Liu, Fellow, IEEE
}

\begin{abstract}
The potential for capacity increase in multiple-antenna wireless communication systems has drawn considerable attention to space-time codes. However, most of the existing space-time code construction methods have assumed ideal channel models: either quasi-static fading or fast fading. In this work, we propose a systematic space-time trellis code construction method for correlated fading channels. We derive the performance criteria that take into account both spatial and temporal channel correlation, assuming that the space-time correlation matrix is of full rank. Moreover, we introduce a new design criterion and analyze the properties of the space-time trellis codes satisfying the proposed criterion. Using the design criterion, we develop a code construction procedure that jointly considers diversity advantage and coding advantage for an arbitrary number of transmit antennas and any memoryless modulation. The flexibility of the proposed code construction method is demonstrated by designing space-time trellis codes for two, three, and four transmit antennas with quaternary phase-shift keying (QPSK), 8PSK (phase-shift keying), and 4ASK (amplitude-shift keying) modulations. The simulation results demonstrate that not only can our method generate new codes for an arbitrary number of antennas, but in some special cases, where previously proposed codes exist, our codes also perform very well.
\end{abstract}

Index Terms-Diversity, space-time codes, space-time correlation, trellis codes, wireless communications.

\section{INTRODUCTION}

In wireless communications, diversity techniques have been used extensively to improve the quality of transmission at high data rates. Spatial diversity corresponds to adding redundancy in the spatial domain: building a system with multiple transmit and/or receive antennas can improve the performance and throughput of the wireless link by making use of the large number of propagation paths between the transmitter and the receiver.

Space-time (ST) trellis codes represent a combination of forward error correction, transmit diversity, and modulation. The performance criteria for both quasi-static channels (the channel stays constant over one frame period) and fast fading channels (the channel changes independently from channel symbol period to channel symbol period) were derived in [1], characterizing the ST codes with two quantities: the diversity advantage, which describes the asymptotic error rate decrease as a function of the signal-to-noise ratio (SNR), and the coding advantage, which determines the vertical shift of the error performance curve.

Most of the existing ST trellis code construction methods have assumed ideal channel models: either quasi-static fading or fast fading, without considering the effect of spatial correlation. For the quasi-static channel model, the authors of [1] proposed design rules for two transmit antennas to achieve the maximum achievable diversity advantage. Later works [2], [3], [12] described systematic code design methods for an arbitrary number of transmit antennas.

Manuscript received May 19, 2003; revised June 11, 2004. The material in this correspondence was presented in part at the IEEE International Conference on Communications, Anchorage, AK, May 2003.

Z. Safar is with the Department of Innovation, IT University of Copenhagen, Copenhagen 2300, Denmark (e-mail: safar@itu.dk).

K. J. R. Liu is with the Department of Electrical and Computer Engineering, University of Maryland, College Park, MD 20742, USA (e-mail: kjrliu@eng.umd.edu).

Communicated by G. Caire, Associate Editor for Communications.

Digital Object Identifier 10.1109/TIT.2004.836926
The first ST trellis code construction method for the fast-fading channel model was described in [4]. ST codes for two transmit antennas and quaternary phase-shift keying (QPSK) modulation were designed using the idea of signal set partitioning. In [5], the design of ST codes for fast-fading channels was also considered. The authors found ST codes for two transmit antennas and QPSK and 8PSK (phase-shift keying) modulations through computer search.

For the quasi-static channel model, the authors of [6] investigated the achievable diversity order as a function of spatial correlation, taking into account some physical propagation parameters. The problem of code design for correlated fading channels was addressed in [7], and general performance criteria were derived for ST-correlated Rayleigh-fading channels. In [8], it was assumed that the channel stays constant for a number of channel symbol periods equal to the number of transmit antennas. The performance criteria were obtained for this channel model, and hand-crafted trellis codes were proposed combining multiple trellis-coded modulation with Alamouti's scheme [9].

In [11], characterizing the performance of ST codes over ST-correlated Rayleigh-fading channels was also considered. The minimum diversity order achievable over all ST correlation matrices of a given rank was defined as the measure of robustness. The relationship between the robustness (diversity) and the rank of the ST correlation matrix was investigated, and upper and lower bounds on the achievable diversity were derived. However, exact results on the achievable diversity advantage and results on the coding advantage were not presented.

In this correspondence, we consider the problem of ST trellis code design, taking into account both spatial and temporal channel correlation. As ad hoc code design methods and computer search methods can only provide point solutions in the design space, and the computational complexity of exhaustive search becomes prohibitive as the number of transmit antennas and the constellation size increase, we focus our attention on the development of systematic ST code design methods that are flexible and scalable.

First, we derive the performance criteria for a channel model in which the channel changes from channel symbol period to channel symbol period in a correlated manner, assuming that the ST correlation matrix is of full rank. We show that for this transmission scenario, the effect of the channel correlation and the ST code on the performance can be separated, resulting in channel-independent performance criteria. Our result implies that as long as the correlation matrix is of full rank, it does not matter what the correlation matrix actually is from the viewpoint of code design, and the ST code design problem for correlated channels can be reduced to the code design problem for fast-fading channels. We characterize the performance of the ST codes by finding exact expressions for the achieved diversity advantage and coding advantage.

Then, we propose a new design criterion and analyze the properties of the ST trellis codes satisfying the proposed criterion. Finally, we develop a systematic code construction procedure that jointly considers diversity advantage and coding advantage for an arbitrary number of transmit antennas and any memoryless modulation. The proposed design method has extremely low complexity: we provide a closed-form solution to the code design problem. To our knowledge, our method is the first systematic ST trellis code design method proposed for nonquasi-static channel models.

\section{SySTEM MODEL AND NOTATION}

Consider a wireless communication system with $K$ transmit and $L$ receive antennas. The input bit stream is divided into $b_{s}$-bit-long 
blocks, forming $B$-ary $\left(B=2^{b_{s}}\right)$ source symbols. At discrete time $t(t=0,1, \ldots, T-1)$, the ST encoder takes the current source symbol $b_{t}\left(b_{t} \in\{0,1, \ldots, B-1\}\right)$ as input and outputs $K B$-ary labels, one for each transmit antenna. We denote the label for antenna $k$ at time $t$ by $i_{t}^{k}\left(i_{t}^{k} \in\{0,1, \ldots, B-1\}\right)$, and the label vector at time $t$ is given by $\boldsymbol{i}_{t}=\left[i_{t}^{0}, i_{t}^{1}, \ldots, i_{t}^{K-1}\right]^{T}$. The labels are mapped onto channel symbols, or constellation points, by the modulators and transmitted through the transmit antennas. In the sequel, $c(i)$ will represent the constellation point corresponding to label $i$ (for example, in case of $B$-ary PSK, $c(i)=\exp (j 2 \pi i / B)$, where $j=\sqrt{-1})$. All the constellations are assumed to be normalized so that the average energy of the constellation is unity (if the channel symbols are equally likely). The channel symbol transmitted by antenna $k$ at time $t$ will be denoted by $c_{t}^{k}=c\left(i_{t}^{k}\right)$, and the vector of channel symbols at time $t$ is defined as $\boldsymbol{c}_{t}=\left[c_{t}^{0}, c_{t}^{1}, \ldots, c_{t}^{K-1}\right]^{T}$.

The transmission medium is assumed to be a flat (frequency-nonselective), ST-correlated Rayleigh-fading channel. The channel coefficient between transmit antenna $k$ and receive antenna $l$ at time $t$ will be denoted by $\alpha_{k, l}(t)$. These channel coefficients are modeled as complex, zero-mean, Gaussian random variables with unit variance and are assumed to be known by the receiver. Based on the above assumptions, after down-conversion, matched filtering, and sampling, $r_{t}^{l}$, the received signal at receive antenna $l$ at discrete time $t$, can be expressed as

$$
r_{t}^{l}=\sum_{k=0}^{K-1} \sqrt{\frac{E_{0}}{K}} \alpha_{k, l}(t) c_{t}^{k}+z_{t}^{l}
$$

where $E_{0}$ is the average transmission energy per source symbol (each transmit antenna transmits with $E_{0} / K$ average transmit energy). The receiver noise, denoted by $z_{t}^{l}$, is taken from samples of independent, complex, zero mean, Gaussian random variables with variance $N_{0}$. The average SNR per source symbol at receive antenna $l$ will be defined as $\mathrm{SNR}_{l}=E_{0} / N_{0}$. To express the received signal in matrix-vector notation, we define the matrix

$$
\boldsymbol{\Gamma}^{k}=\operatorname{diag}\left(c_{0}^{k}, c_{1}^{k}, \ldots, c_{T-1}^{k}\right)
$$

and the row vectors

and

$$
\begin{aligned}
\boldsymbol{r}^{l} & =\left[r_{0}^{l}, r_{1}^{l}, \ldots, r_{T-1}^{l}\right] \\
\alpha_{k, l} & =\left[\alpha_{k, l}(0), \alpha_{k, l}(1), \ldots, \alpha_{k, l}(T-1)\right]
\end{aligned}
$$

$$
\boldsymbol{z}^{l}=\left[z_{0}^{l}, z_{1}^{l}, \ldots, z_{T-1}^{l}\right]
$$

Using these quantities, the $L T$ by 1 received signal vector $\boldsymbol{r}=\left[\boldsymbol{r}^{0}, \boldsymbol{r}^{1}, \ldots, \boldsymbol{r}^{L-1}\right]^{T}$ is given by

$$
\boldsymbol{r}=\sqrt{\frac{E_{0}}{K}} \boldsymbol{\Gamma} \alpha+z
$$

with the $L T$ by $K L T$ channel symbol matrix

$$
\Gamma=\boldsymbol{I}_{L} \otimes\left[\boldsymbol{\Gamma}^{0}, \boldsymbol{\Gamma}^{1}, \ldots, \Gamma^{K-1}\right]
$$

the $L T$ by 1 noise vector

$$
\boldsymbol{z}=\left[\boldsymbol{z}^{0}, \boldsymbol{z}^{1}, \ldots, \boldsymbol{z}^{L-1}\right]^{T}
$$

and the $K L T$ by 1 channel coefficient vector

$$
\alpha=\left[\alpha_{0,0}, \alpha_{1,0}, \ldots, \alpha_{K-1,0}, \alpha_{0,1}, \ldots, \alpha_{K-1, L-1}\right]^{T} .
$$

The symbol $\otimes$ denotes the tensor product, and $\boldsymbol{I}_{L}$ is the $L$ by $L$ identity matrix. The correlation matrix $R=E\left(\alpha \alpha^{H}\right)$ has $K L T$ rows and $K L T$ columns and is assumed to be of full rank (i.e., its eigenvalues are real and positive).

Due to decoding errors, the receiver may decode a different sequence of channel symbols. The erroneously decoded channel symbol for transmit antenna $k$ at time $t$ will be denoted by $\hat{c}_{t}^{k}$, and the vector of decoded channel symbols at time $t$ will be given by

$$
\hat{\boldsymbol{c}}_{t}=\left[\hat{c}_{t}^{0}, \hat{c}_{t}^{1}, \ldots, \hat{c}_{t}^{K-1}\right]^{T} .
$$

Defining the matrix

$$
\hat{\Gamma}^{k}=\operatorname{diag}\left(\hat{c}_{0}^{k}, \hat{c}_{1}^{k}, \ldots, \hat{c}_{T-1}^{k}\right)
$$

the erroneously decoded channel symbol matrix $\hat{\Gamma}$ can be expressed similarly to $\boldsymbol{\Gamma}$.

\section{PERformance CRITERIA}

In this section, we derive the performance criteria for ST-correlated Rayleigh-fading channels with full-rank correlation matrix, based on an upper bound on the pairwise error probability [7], derived for a general transmission scenario. It was shown in [7] that the probability that the maximum-likelihood decoder erroneously decodes the channel symbol matrix $\hat{\Gamma}$ if $\Gamma$ was sent can be upper-bounded as

$$
P(\hat{\boldsymbol{\Gamma}} \mid \boldsymbol{\Gamma}) \leq \frac{\left(\begin{array}{c}
2 r-1 \\
r-1
\end{array}\right)\left(\frac{E_{0}}{K N_{0}}\right)^{-r}}{\Pi_{i=1}^{r} \gamma_{i}}
$$

where $r$ and $\gamma_{i}$ 's are the rank and the nonzero eigenvalues of the matrix $\boldsymbol{\Delta} \boldsymbol{R} \boldsymbol{\Delta}^{H}$, respectively, and $\boldsymbol{\Delta}$ is the channel symbol difference matrix, defined as $\boldsymbol{\Delta}=\boldsymbol{\Gamma}-\hat{\boldsymbol{\Gamma}}$. The performance criteria are obtained by simplifying (3) when the received signal is described by (2).

Assume that for $\tau$ time instants $t_{0}, t_{1}, \ldots, t_{\tau-1}$, the sent and the erroneously decoded channel symbol vectors are different, i.e., $\boldsymbol{c}_{t}-$ $\hat{c}_{t} \neq \mathbf{0}$ for $t \in\left\{t_{0}, t_{1}, \ldots, t_{\tau-1}\right\}$, and for the rest of the time instants, they are the same. Therefore, the sent and decoded channel symbol vectors corresponding to the times $t \notin\left\{t_{0}, t_{1}, \ldots, t_{\tau-1}\right\}$ will produce all-zero rows and columns in the channel symbol difference matrix $\Delta$. These rows and columns can be eliminated from the analysis in the following way. For each $t \notin\left\{t_{0}, t_{1}, \ldots, t_{\tau-1}\right\}$, rows $t$, $t+T, t+2 T, \ldots, t+(L-1) T$ and columns $t, t+T, t+2 T, \ldots$, $t+(K L-1) T$ are removed from the matrix $\Delta$, producing a new $L \tau$ by $K L \tau$ channel symbol difference matrix $\Delta^{\prime}$. The matrix $\Delta^{\prime}$ has a structure similar to $\Gamma$, but the matrices $\Gamma^{k}$ are replaced with

$$
\boldsymbol{\Delta}^{\prime k}=\operatorname{diag}\left(c_{t_{0}}^{k}-\hat{c}_{t_{0}}^{k}, c_{t_{1}}^{k}-\hat{c}_{t_{1}}^{k}, \ldots, c_{t_{\tau-1}}^{k}-\hat{c}_{t_{\tau-1}}^{k}\right) .
$$

Note that $\Delta^{\prime}$ has full row rank.

In addition, for each $t \notin\left\{t_{0}, t_{1}, \ldots, t_{\tau-1}\right\}$, rows and columns $t$, $t+T, t+2 T, \ldots, t+(K L-1) T$ must also be removed from $\boldsymbol{R}$, resulting in the $K L \tau$ by $K L \tau$ matrix $\boldsymbol{R}^{\prime}$. Since only all-zero rows and columns have been deleted from $\boldsymbol{\Delta}$, the nonzero eigenvalues of $\boldsymbol{\Delta} \boldsymbol{R} \boldsymbol{\Delta}^{H}$ and $\boldsymbol{\Delta}^{\prime} \boldsymbol{R}^{\prime} \boldsymbol{\Delta}^{\prime H}$ are the same. It is shown in Appendix $A$ that the relation

$$
\prod_{i=1}^{r} \gamma_{i}=\operatorname{det}\left(\boldsymbol{\Delta}^{\prime} \boldsymbol{R}^{\prime} \boldsymbol{\Delta}^{\prime H}\right) \geq \operatorname{det}\left(\boldsymbol{\Lambda}_{\min }(L \tau)\right) \operatorname{det}\left(\boldsymbol{\Delta}^{\prime} \boldsymbol{\Delta}^{\prime H}\right)
$$

holds, where $\Lambda_{\min }(L \tau)$ is a $L \tau$ by $L \tau$ diagonal matrix with the $L \tau$ smallest eigenvalues of $\boldsymbol{R}$ along the diagonal. Since $\boldsymbol{R}$ is positive definite, $\operatorname{det}\left(\boldsymbol{\Lambda}_{\min }(L \tau)\right)$ is strictly positive. Moreover, $\boldsymbol{\Delta}^{\prime}$ has full row 
rank, so $\operatorname{det}\left(\boldsymbol{\Delta}^{\prime} \boldsymbol{\Delta}^{\prime H}\right)$ is also strictly positive. Consequently, the matrices $\boldsymbol{\Delta} \boldsymbol{R} \boldsymbol{\Delta}^{H}$ and $\boldsymbol{\Delta}^{\prime} \boldsymbol{R}^{\prime} \boldsymbol{\Delta}^{\prime H}$ are both of rank $L \tau$. Combining (3) with (4), and recognizing that

$$
\operatorname{det}\left(\boldsymbol{\Delta}^{\prime} \boldsymbol{\Delta}^{\prime H}\right)=\prod_{i=0}^{\tau-1}\left\|\boldsymbol{c}_{t_{i}}-\hat{\boldsymbol{c}}_{t_{i}}\right\|^{2 L}
$$

where $\|x\|=\sqrt{\boldsymbol{x}^{H} \boldsymbol{x}}$, we arrive at the upper bound

$$
\begin{aligned}
P(\hat{\Gamma} \mid \mathbf{\Gamma}) \leq\left(\frac{E_{0}}{K N_{0}}\right)^{-L \tau} & \left(\begin{array}{c}
2 L \tau-1 \\
L \tau-1
\end{array}\right) \\
& \times \frac{1}{\operatorname{det}\left(\boldsymbol{\Lambda}_{\min }(L \tau)\right)} \prod_{i=0}^{\tau-1}\left\|\boldsymbol{c}_{t_{i}}-\hat{\boldsymbol{c}}_{t_{i}}\right\|^{-2 L}
\end{aligned}
$$

The performance criteria now can be formulated to minimize the maximum value of $P(\hat{\boldsymbol{\Gamma}} \mid \mathbf{\Gamma})$.

1) Design for diversity advantage (distance criterion): The minimum number of time instants when the correct and the decoded channel symbol vectors are different (the minimum value of $\tau$ ) taken over all possible correct and erroneously decoded channel symbol vector sequences must be maximized.

2) Design for coding advantage (product criterion): The minimum of the norm products, defined as

$$
\delta=\prod_{i=0}^{\tau-1}\left\|\boldsymbol{c}_{t_{i}}-\hat{\boldsymbol{c}}_{t_{i}}\right\|^{2}
$$

taken over all possible correct and erroneously decoded channel symbol vector sequences must be maximized.

Note that the these performance criteria are the same as the performance criteria proposed for fast (independently) fading channels [1]. In case of independent fading, the matrices $R, R^{\prime}$, and $\boldsymbol{\Lambda}_{\text {min }}(L \tau)$ become identity matrices, and (5) simplifies to a form similar to the upper bound derived in [1], the only difference being a multiplicative constant.

In the above derivation, the matrix $\boldsymbol{R}$ was assumed to have full rank. This corresponds to the condition that the magnitude of the channel correlation decay fast enough as the transmit and receive antenna separation and the time separation increase. If this condition holds, the code design problem for ST-correlated channels can be reduced to the code design problem for fast fading channels. Moreover, the correlation only causes coding advantage loss compared to the fast (independent) fading case.

\section{A DESIGN CRITERION FOR TRELLIS CODES}

This section proposes a new design criterion that is based on the distance criterion described in the previous section. Since the design criterion is specific to trellis codes, it is necessary to extend the notation to explicitly show the dependence of the channel symbols on the state transitions.

The ST trellis encoder works as a finite-state machine with $N$ states: at discrete time $t$, it takes the current source symbol $b_{t}$, and governed by this input and the current state, $S_{t}\left(S_{t} \in\{0,1, \ldots, N-1\}\right)$, it moves to the next state $S_{t+1}$. The label for transmit antenna $k$ produced by the encoder during the state transition from $S_{t}$ through the branch corresponding to $b_{t}$ will be denoted by $i^{k}\left(S_{t}, b_{t}\right)=i_{t}^{k}$. The label vector $\boldsymbol{i}_{t}$ will become

$$
\boldsymbol{i}\left(S_{t}, b_{t}\right)=\left[i^{0}\left(S_{t}, b_{t}\right), \ldots, i^{K-1}\left(S_{t}, b_{t}\right)\right]^{T}
$$

and the channel symbol vector $c_{t}$ will be written as

$$
c\left(S_{t}, b_{t}\right)=\left[c\left(i^{0}\left(S_{t}, b_{t}\right)\right), \ldots, c\left(i^{K-1}\left(S_{t}, b_{t}\right)\right)\right]^{T} .
$$

Similarly, $c\left(\hat{S}_{t}, \hat{b}_{t}\right)=\hat{\boldsymbol{c}}_{t}$ will be the erroneously decoded channel symbol vector at time $t$. This notation emphasizes that, in general, the state sequences corresponding to the correct and the erroneously decoded paths (i.e., $\left\{S_{t}\right\}$ and $\left\{\hat{S}_{t}\right\}$ ) are different, and so are the encoded source symbol sequence $\left\{b_{t}\right\}$ and the decoded source symbol sequence $\left\{\hat{b}_{t}\right\}$. The design criterion was developed for encoders having $N_{\text {min }}=B^{K-1}$ states, so from now on it will be assumed that the encoder has $N_{\min }$ states.

The proposed design criterion (uniqueness criterion) is: Every label vector must be unique. This means that a) the label vectors assigned to different branches emanating from the same state must be different, and b) any label vector assigned to any branch emanating from any state must be different from any label vector assigned to any branch emanating from any other state.

Each label vector contains $K B$-ary labels, so there are $B^{K}$ different label vectors. Since there are $B$ branches emanating from each state and the encoder is assumed to have $N_{\text {min }}$ states, we need exactly $B^{K}$ different label vectors. Therefore, it is possible to assign label vectors to state transitions according to the uniqueness criterion. Sections IV-A and -B will analyze the properties of the ST codes that satisfy the proposed uniqueness criterion.

\section{A. Diversity Advantage}

Assume that the first decoding error occurs at $t=t_{0}$, so the correct and decoded paths diverge at this point (i.e., $S_{t_{0}}=\hat{S}_{t_{0}}$ and $b_{t_{0}} \neq \hat{b}_{t_{0}}$ ). The two paths are assumed to merge later at time $t=t_{0}+\tau$, resulting in a $\tau$-length error event. As a consequence of criterion a), the channel symbol vectors corresponding to the two paths diverging at $S_{t_{0}}$ will be different. Moreover, as a result of criterion b), the channel symbol vectors corresponding to the correct and the decoded paths going through different states will be different. Therefore, for $t=$ $t_{0}, t_{0}+1, \ldots, t_{0}+\tau-1$, we have $c\left(S_{t}, b_{t}\right)-c\left(\hat{S}_{t}, \hat{b}_{t}\right) \neq 0$, so an error event of length $\tau$ will always achieve a Hamming distance $\tau$. (Here we define the Hamming distance between the sent and decoded codewords as the number of discrete time instants, or positions, where the sent and the decoded channel symbol vectors differ in at least one coordinate.)

From the performance criteria described in Section III, one can conclude that any $\tau$-length error event will achieve a diversity advantage of $\tau L$. In case of multiple error events, the total diversity advantage will be the sum of the diversity advantages of the individual error events. Consequently, the minimum diversity advantage of the ST code is determined by the shortest error event. For ST encoders having $N_{\text {min }}$ states, the shortest error event is $K$ state transitions long [12], so the ST code is guaranteed to achieve a diversity advantage of $K L$.

\section{B. Error Event Probability}

In this section, we will derive an upper bound on the $\tau$-length error event probability, i.e., the probability that at a given discrete time instant, a decoding error occurs, resulting in an error event that is $\tau$ state transitions long.

Using the union bound, the $\tau$-length error event probability, which is the probability of the union of the $\tau$-length error events, can be upperbounded as

$$
P_{\tau} \leq \sum_{\boldsymbol{\Gamma}} \sum_{\hat{\mathbf{\Gamma}}_{\tau}} P\left(\hat{\boldsymbol{\Gamma}}_{\tau} \mid \boldsymbol{\Gamma}\right) P(\boldsymbol{\Gamma})
$$


In (6), the first summation is over all possible sent channel symbol matrices $\boldsymbol{\Gamma}$, and the second summation is over all possible erroneously decoded channel symbol matrices $\hat{\boldsymbol{\Gamma}}_{\tau}$ corresponding to $\tau$-length error events for a particular $\boldsymbol{\Gamma}$. Assuming that the decoding error occurs at time $t_{0}$, for $t=t_{0}, t_{0}+1, \ldots, t_{0}+\tau-1$, at least one coordinate of the channel symbol vectors $c\left(S_{t}, b_{t}\right)$ and $\boldsymbol{c}\left(\hat{S}_{t}, \hat{b}_{t}\right)$ will be different. Therefore, if we denote the minimum distance of the chosen constellation by $d$, the squared norms of their differences can be lower-bounded as

$$
\left\|\boldsymbol{c}\left(S_{t}, b_{t}\right)-\boldsymbol{c}\left(\hat{S}_{t}, \hat{b}_{t}\right)\right\|^{2} \geq d^{2}
$$

for $t=t_{0}, t_{0}+1, \ldots, t_{0}+\tau-1$. Combining (5) with (7) and using the inequality

$$
\left(\begin{array}{c}
2 n-1 \\
n-1
\end{array}\right)<4^{n}
$$

we obtain an upper bound on the pairwise error probability

$$
P\left(\hat{\boldsymbol{\Gamma}}_{\tau} \mid \boldsymbol{\Gamma}\right)<\frac{1}{\operatorname{det}\left(\boldsymbol{\Lambda}_{\min }(L \tau)\right)}\left(\frac{E_{0}}{4 K N_{0}}\right)^{-L \tau} d^{-2 L \tau}
$$

Defining $\gamma_{\text {min }}$, the minimum determinant taken over all possible $\tau$ values, as

$$
\gamma_{\min }=\min _{1 \leq \tau \leq T-1} \operatorname{det}\left(\Lambda_{\min }(L \tau)\right)
$$

the pairwise error probability can be further upper-bounded as

$$
P\left(\hat{\boldsymbol{\Gamma}}_{\tau} \mid \mathbf{\Gamma}\right)<\frac{1}{\gamma_{\min }}\left(\frac{E_{0}}{4 K N_{0}}\right)^{-L \tau} d^{-2 L \tau}
$$

Since the upper bound (10) does not depend on $\hat{\boldsymbol{\Gamma}}_{\tau},(6)$ can be rewritten as

$$
P_{\tau}<\frac{1}{\gamma_{\min }} \sum_{\boldsymbol{\Gamma}} N\left(\hat{\boldsymbol{\Gamma}}_{\tau}\right)\left(\frac{E_{0} d^{2}}{4 K N_{0}}\right)^{-L \tau} P(\boldsymbol{\Gamma})
$$

where $N\left(\hat{\boldsymbol{\Gamma}}_{\tau}\right)$ is the number of $\tau$-length error paths for a given correct path. $N\left(\hat{\boldsymbol{\Gamma}}_{\tau}\right)$ can be strictly upper-bounded by $B^{\tau}$, so the upper bound simplifies to

$$
P_{\tau}<\frac{1}{\gamma_{\min }} \sum_{\boldsymbol{\Gamma}} B^{\tau}\left(\frac{E_{0} d^{2}}{4 K N_{0}}\right)^{-L \tau} P(\boldsymbol{\Gamma})
$$

Finally, recognizing that $P(\boldsymbol{\Gamma})$ is the only term that depends on $\Gamma$, and that the probability mass function of $\Gamma$ sums to unity, the final expression for the upper bound on the $\tau$-length error event probability becomes

$$
P_{\tau}<\frac{1}{\gamma_{\min }}\left(\frac{E_{0} d^{2}}{4 K N_{0} \sqrt[L]{B}}\right)^{-L \tau}
$$

From (13), one can see that the probability that an error event of a given length occurs decreases at least exponentially with its length. Consequently, at high SNR, the shortest error events will dominate; the longer error paths will have negligible contribution to the error event probability. (Note that we assumed that the trellis is finite, so the range of the $\tau$ values is constrained by the length of the trellis, the time when the decoding error occurs, and the length of the shortest error event.)

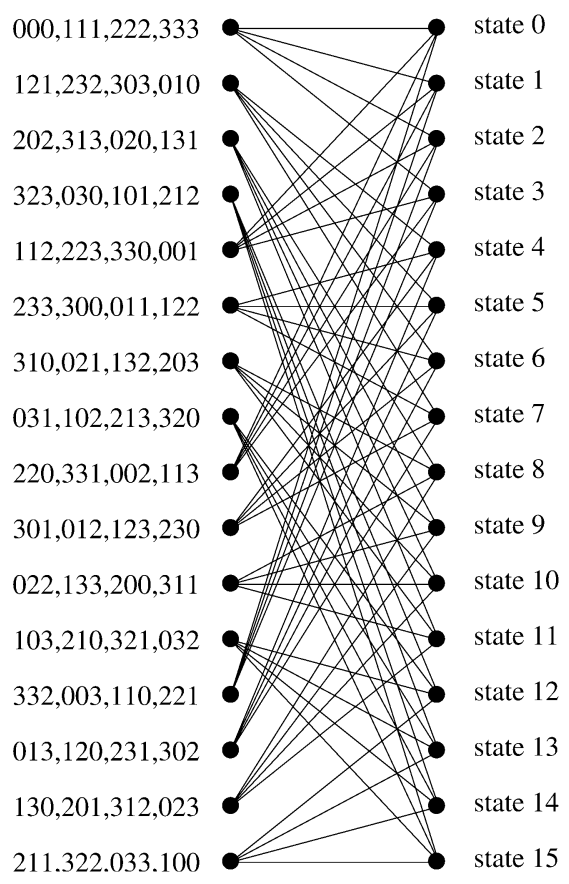

Fig. 1. Example ST code for three antennas, 4-ary modulation.

\section{The Design Method}

In the ST encoder, $B$ label vectors are assigned to each state, according to the branches emanating from that state. The current source symbol selects one of them, and the $k$ th $(k=0,1, \ldots, K-1)$ coordinate of the chosen vector determines the constellation point for antenna $k$. Fig. 1 depicts an example ST code for three antennas and any 4 -ary constellation $(K=3, B=4, N=16)$. In this case, if the current state is state 2 and the value of the current source symbol is 3 , the ST encoder selects the third label vector $[1,3,1]^{T}$ and moves to state 11 . The zeroth, first, and second antennas will transmit the channel symbols corresponding to the labels 1,3 , and 1 , respectively. Note that the $\mathrm{ST}$ encoder is assumed to have $N_{\min }=B^{K-1}$ states, so any state $S$ $\left(S \in\left\{0,1, \ldots, B^{K-1}-1\right\}\right)$ can be uniquely represented as a $(K-1)$ digit $B$-ary number with digits $l_{1}, l_{2}, \ldots, l_{K-1}\left(l_{k} \in\{0,1, \ldots, B-1\}\right)$

$$
S=B^{K-2} l_{K-1}+B^{K-3} l_{K-2}+\cdots+B l_{2}+l_{1} .
$$

This section addresses the problem of assigning label vectors to state transitions. As we have seen in Section IV, the ST codes satisfying the uniqueness criterion posses some very advantageous properties: they can deliver the maximum diversity available in the channel model for the given number of encoder states, and the error event probability decays exponentially with the the length of the error event. Thus, we require that the designed ST codes satisfy the uniqueness criterion. However, this design criterion does not specify the ST codes uniquely (the available $B^{K}$ label vectors can be arranged in $\left(B^{K}\right)$ ! different ways), so the remaining freedom can be used to increase the value of the minimum norm product $(\delta)$. Now we present a method that provides a closed-form solution to the code design problem. The basic idea behind the method is that it attempts to maximize the minimum norm product corresponding to the shortest error events by maximizing the number of nonzero coordinates in the channel symbol difference vectors. Consequently, the code design method is modulation independent, so it can be used with any memoryless modulation.

The proposed design rules for ST trellis codes for $K$ transmit antennas and $N_{\min }$ encoder states are as follows. 
1) The 0th coordinate of the label vector (the label for the 0th transmit antenna) corresponding to branch $b(b \in\{0,1, \ldots, B-1\})$ emanating from state $S\left(S \in\left\{0,1, \ldots, B^{K-1}-1\right\}\right)$ is determined as

$$
i^{0}(S, b)=\left(b+l_{K-1}+l_{K-2}+\cdots+l_{1}\right) \bmod B
$$

where $l_{K-1}, l_{K-2}, \ldots, l_{1}$ are the digits of the $B$-ary representation of state $S$ in (14).

2) For $k=1,2, \ldots, K-1$, the $k$ th coordinate of the label vector (the label for the $k$ th transmit antenna) corresponding to branch $b$ emanating from state $S$ is calculated as

$$
i^{k}(S, b)=\left(i^{0}(S, b)+l_{k}\right) \bmod B .
$$

By making use of the identity

$$
\left(\sum_{i=1}^{n} \alpha_{i} \bmod \beta\right) \bmod \beta=\left(\sum_{i=1}^{n} \alpha_{i}\right) \bmod \beta
$$

the second design rule can be put in the alternative form

$$
i^{k}(S, b)=\left(b+l_{K-1}+l_{K-2}+\cdots+l_{1}+l_{k}\right) \bmod B .
$$

As an example, consider the ST code shown in Fig. 1. Since $B=4$, state $S=6$ can be represented as $S=B l_{2}+l_{1}$ with $l_{2}=1$ and $l_{1}=2$. The labels corresponding to the second branch $(b=2)$ emanating from state 6 are determined as

$$
\begin{aligned}
& i^{0}(S, b)=\left(b+l_{2}+l_{1}\right) \bmod B=1 \\
& i^{1}(S, b)=\left(i^{0}(S, b)+l_{1}\right) \bmod B=3
\end{aligned}
$$

and

$$
i^{2}(S, b)=\left(i^{0}(S, b)+l_{2}\right) \bmod B=2 .
$$

Therefore, the label vector assigned to this state transition will be $[1,3,2]^{T}$.

From the above design rules, it is not apparent that the resulting ST codes satisfy the uniqueness criterion. The following result will establish that the uniqueness criterion is indeed satisfied.

Theorem 1: The ST trellis codes produced by the above described construction method for $K$ transmit antennas and $N_{\min }$ states satisfy the uniqueness criterion with any $B$-ary constellation.

Proof: The proof is given in two steps.

1) From (15), it can be seen that for a given state $S$ (given $l_{1}, l_{2}, \ldots, l_{K-1}$ values) and different $b$ input values, the value of $i^{0}(S, b)$ will be different. Therefore, the label vectors assigned to the same state are different in at least one (the 0th) coordinate.

2) Let us pick two arbitrary states, $S_{1}$ and $S_{2}\left(S_{1} \neq S_{2}\right)$, and one label vector assigned to a branch emanating from each state. If the 0th coordinates of the label vectors are different, then the statement is proven. If they are the same, then we have $i^{0}\left(S_{1}, b_{1}\right)=i^{0}\left(S_{2}, b_{2}\right)$ for some $b_{1}, b_{2} \in\{0,1, \ldots, B-1\}$. The states $S_{1}$ and $S_{2}$ can be uniquely expressed as

$$
\begin{aligned}
& S_{1}=B^{K-2} l_{K-1}+B^{K-3} l_{K-2}+\cdots+B l_{2}+l_{1} \\
& S_{2}=B^{K-2} m_{K-1}+B^{K-3} m_{K-2}+\cdots+B m_{2}+m_{1}
\end{aligned}
$$

with $l_{k}, m_{k} \in\{0,1, \ldots, B-1\} . S_{1} \neq S_{2}$, so $l_{k} \neq m_{k}$ must hold for at least one $k$ value. Since the $k$ th $(k=1,2, \ldots, K-1)$ coordinates of the label vectors are determined according to (16), we will have $i^{k}\left(S_{1}, b_{1}\right) \neq i^{k}\left(S_{2}, b_{2}\right)$ for at least one $k$ value. Therefore, the label vectors assigned to different states are different in at least one coordinate. Both conditions a) and b) of Section IV are satisfied, so the code is guaranteed to satisfy the uniqueness criterion.
As it is well known from the literature [1], [8], an ST code designed for a particular channel model may suffer considerable performance loss in case of a different channel model. Therefore, it is of interest to investigate how the performance of the proposed ST codes is affected when transmitting them over very slowly fading channels. The following theorem characterizes the performance of the two-antenna PSK ST trellis codes designed by the proposed code construction procedure in the case of the quasi-static fading channel model. It states that these codes also achieve full (spatial) diversity in quasi-static fading environment.

Theorem 2: The ST trellis codes designed by the proposed method for two transmit antennas and $B$-ary PSK modulation satisfy the rank criterion [1] derived for quasi-static fading channels.

Proof: Assume that the correct and the decoded paths diverge at state $S_{0}$ (i.e., $S_{0}=\hat{S}_{0}$ and $b_{0} \neq \hat{b}_{0}$ ). Since the shortest error event is $K$ state transitions long, the two paths can only merge at state $S_{K}$ or later. Assuming that the two paths merge at state $S_{T+K}$ for some $T \geq 0$ and considering the $K=2$ case, we have $S_{T+2}=\hat{S}_{T+2}$. The basic idea behind the proof is to show that the channel symbol difference vectors corresponding to $t=0$ (when the two paths diverge) and $t=T+2$ (when the two paths merge) form a linearly independent set. We will do this by proving that the code difference matrix $D_{2}$, defined as

$$
\boldsymbol{D}_{2}=\left[\begin{array}{ll}
d_{0}^{0} & d_{T+2}^{0} \\
d_{0}^{1} & d_{T+2}^{1}
\end{array}\right]
$$

where $d_{t}^{k}=c\left(i^{k}\left(S_{t}, b_{t}\right)\right)-c\left(i^{k}\left(\hat{S}_{t}, \hat{b}_{t}\right)\right)$, is of full rank for all possible $(T+2)$-length correct and erroneously decoded paths. The first column of $D_{2}$ can be determined easily, recognizing that the two-antenna ST encoder has $B$ states and expressing the first erroneously decoded source symbol $\hat{b}_{0}$ as $\hat{b}_{0}=\left(b_{0}+\Delta_{b}\right) \bmod B$ for some $\Delta_{b} \in\{1,2, \ldots, B-1\}$. Using design rules (15), (18), and the identity (17), and assuming $B$-ary PSK modulation, we obtain

$$
\begin{aligned}
& d_{0}^{0}=e^{j \frac{2 \pi}{B}\left(S_{0}+b_{0}\right)}\left(1-e^{j \frac{2 \pi}{B} \Delta_{b}}\right) \\
& d_{0}^{1}=e^{j \frac{2 \pi}{B}\left(2 S_{0}+b_{0}\right)}\left(1-e^{j \frac{2 \pi}{B} \Delta_{b}}\right) .
\end{aligned}
$$

Note that the modulo operation can be omitted because of the periodicity of the complex exponentiation. The second column of $\boldsymbol{D}_{2}$ is determined as follows. It is shown in Appendix B that for encoders having $N_{\text {min }}$ states, the state transition $S_{T+K}, T \geq 0$, can be expressed as

$$
S_{T+K}=\sum_{m=T+1}^{T+K-1} B^{T+K-1-m} b_{m} .
$$

For $K=2$, (21) simplifies to $S_{T+2}=b_{T+1}$. Since the correct and the erroneous paths merge at $S_{T+2}$, we have

$$
S_{T+2}=b_{T+1}=\hat{S}_{T+2}=\hat{b}_{T+1} .
$$

Moreover, the two paths do not merge before time $T+2$, so state $\hat{S}_{T+1}$ can be written as $\hat{S}_{T+1}=\left(S_{T+1}+\Delta_{S}\right) \bmod B$ for some $\Delta_{S} \in$ $\{1,2, \ldots, B-1\}$. As a result, applying design rules (15), (18), and the identity (17), and assuming $B$-ary PSK modulation, we arrive at

$$
\begin{aligned}
& d_{T+2}^{0}=e^{j \frac{2 \pi}{B}\left(S_{T+1}+b_{T+1}\right)}\left(1-e^{j \frac{2 \pi}{B} \Delta_{S}}\right) \\
& d_{T+2}^{1}=e^{j \frac{2 \pi}{B}\left(2 S_{T+1}+b_{T+1}\right)}\left(1-e^{j \frac{2 \pi}{B} 2 \Delta_{S}}\right) .
\end{aligned}
$$

The matrix $\boldsymbol{D}_{2}$ is rank deficient if and only if its determinant is zero, i.e.,

$$
d_{0}^{0} d_{T+2}^{1}=d_{0}^{1} d_{T+2}^{0} .
$$


TABLE I

Minimum DeterminANT VALUES

\begin{tabular}{|c||c|c|c|}
\hline $\begin{array}{c}\text { number of } \\
\text { TX antennas }\end{array}$ & 2 & 3 & 4 \\
\hline \hline BPSK & 16 & 64 & 256 \\
\hline QPSK & 4 & 8 & 16 \\
\hline 8PSK & 0.3431 & 0.0345 & 0.0035 \\
\hline 16PSK & 0.0232 & $2.4 \cdot 10^{-5}$ & - \\
\hline 4ASK & 0.6400 & 0.5120 & 0.4096 \\
\hline
\end{tabular}

Substituting (20) and (22) into (23) and simplifying the expression, the zero determinant condition becomes

$$
e^{j \frac{2 \pi}{B} S_{T+1}}\left(1-e^{j \frac{2 \pi}{B} 2 \Delta_{S}}\right)=e^{j \frac{2 \pi}{B} S_{0}}\left(1-e^{j \frac{2 \pi}{B} \Delta_{S}}\right)
$$

After taking squared magnitudes of both sides of (24) and simplification, we obtain

$$
\cos \left(\frac{2 \pi}{B} 2 \Delta_{S}\right)=\cos \left(\frac{2 \pi}{B} \Delta_{S}\right) .
$$

Since $B$ is a power of 2 and $\Delta_{S}$ takes on values from the set $\{1,2, \ldots, B-1\}$, the two sides of (25) cannot be equal. Therefore, the determinant of $\boldsymbol{D}_{2}$ cannot be zero, and the ST code satisfies the rank criterion.

We have not been able to prove this property for an arbitrary number of transmit antennas and any memoryless modulation, but we have verified it using computer simulations for some ST codes constructed by our method. Table I shows the minimum determinant values of the ST codes designed for two, three, and four transmit antennas, and BPSK, QPSK, 8PSK, 16PSK, and 4ASK constellations. Since the values of the minimum determinants decay fast with the constellation size, the ST codes designed by previously existing methods [1]-[3], [10], [12] will outperform the proposed ST codes in quasi-static fading environment. However, Theorem 2 and Table I suggest that if a temporally evolving channel becomes constant for a short time period (for example, a vehicle stops at a red light), the proposed ST codes are still able to deliver the available (in this case only spatial) diversity.

\section{DISCUSSION}

In order to achieve a diversity advantage of $K L$, there must be at least $K$ time instants when the sent and the decoded channel symbol vectors are different. Thus, the shortest error event must be at least $K$ state transitions long. This means that for a $B$-ary modulation ( $B$ branches emanating from each state), the encoder must have at least $N_{\text {min }}=B^{K-1}$ states. Consequently, our ST codes achieve the desired diversity level with the minimum possible trellis complexity.

Since the diversity does not depend on the dimensionality of the channel symbol vectors, it is possible to design ST codes that can achieve a diversity advantage of $K L$ with encoders having less than $K$ transmit antennas. However, our design criterion provides extra performance gain in addition to the achieved minimum diversity level. The uniqueness criterion guarantees that the probability of the error events decreases exponentially with their lengths. For an ST code that does not satisfy this criterion, it is possible that the probability of a very long error event (many bit errors) and a very short error event (a few bit errors) are in the same order of magnitude, causing serious performance loss. On the other hand, the uniqueness criterion ensures that the probability of the long error events decays much faster than the probability of the short error events, as the SNR increases. From Section IV, it can be seen that for encoders having $N_{\text {min }}$ states, in order to satisfy the uniqueness criterion, the channel symbol vectors

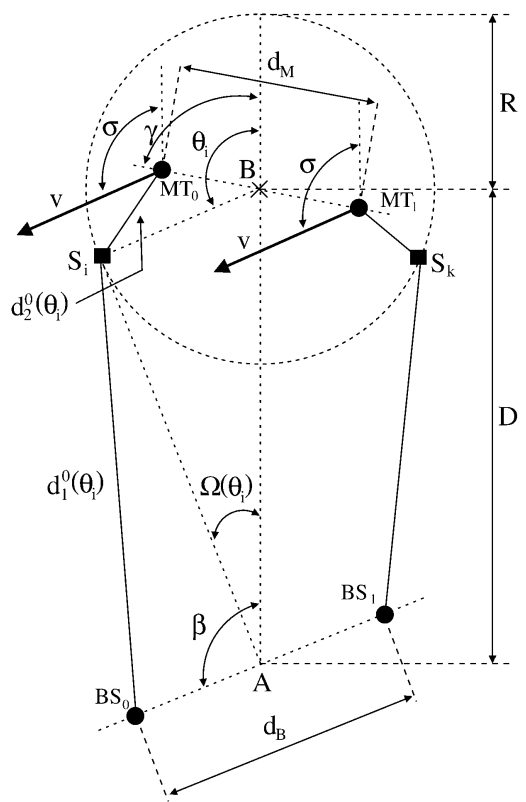

Fig. 2. Geometric model for correlated fading simulations.

must have at least $K$ coordinates, so the encoder must have at least $K$ transmit antennas.

\section{SimUlATION RESULTS}

To illustrate the performance of the codes obtained by the proposed method, we present some simulation results. The source symbols were transmitted in frames of length 130 , and the Viterbi algorithm with decoding depth of 20 state transitions was used to decode the received signals. For the fast-fading channel model, the path gains between the transmit and the receive antennas were independent, complex, zeromean, Gaussian random variables with unit variance at each discrete time instant.

In the correlated fading case, the path gains were generated according to the statistical model described in [13]. The base station (BS) was the transmitter and the mobile terminal (MT) was the receiver. Both the BS and the MT were assumed to have a uniform, linear array of isotropic antennas, and the MT was surrounded by a ring of scatterers. The model parameters were $d_{B}-\mathrm{BS}$ antenna separation, $d_{M}$ - MT antenna separation, $D$-distance between the BS and the MT, $R$-radius of the scatterer ring, $N_{s}$-number of scatterers, $\beta$-direction of the BS antenna array, $\gamma$-direction of the MT antenna array, $\sigma$-direction of the MT movement, $v$-the magnitude of the MT speed, $f_{c}$ - the carrier frequency (or $\lambda_{c}$-the carrier wavelength), and $T_{s}$-the channel symbol period. The geometry of the model is shown in Fig. 2 for two adjacent $\mathrm{BS}$ antennas $\left(\mathrm{BS}_{0}, \mathrm{BS}_{1}\right)$ and two adjacent MT antennas $\left(\mathrm{MT}_{0}, \mathrm{MT}_{1}\right)$. The $i$ th $\left(i=0,1, \ldots, N_{s}-1\right)$ scatterer $S_{i}$ was at an angle $\theta_{i}$ from the middle point of the MT antenna array, and the scatterer angles were uniformly distributed in the range $[-\pi, \pi]$. During the simulations, we used the following parameter values: $D=1 \mathrm{~km}, R=20 \mathrm{~m}, N_{s}=20, \beta=\frac{3 \pi}{4}$ radians, $\gamma=\frac{\pi}{4}$ radians, $\sigma=\frac{3 \pi}{4}$ radians, and $v=70 \mathrm{~km} / \mathrm{h}$. Three cases were considered: a) high correlation $\left(T_{s}=50 \mu \mathrm{s}, f_{c}=900 \mathrm{MHz}\right.$, $\left.d_{B}=5 \lambda_{c}, d_{M}=0.6 \lambda_{c}\right)$; b) low correlation $\left(T_{s}=500 \mu \mathrm{s}, f_{c}=\right.$ $2 \mathrm{GHz}, d_{B}=25 \lambda_{c}, d_{M}=5 \lambda_{c}$ ); and c) no correlation (fast fading).

We present probability of bit error curves as functions of the average SNR per source symbol at the receive antennas. Fig. 3 depicts the performance of the ST code designed by our method for two transmit antennas and QPSK constellation $(K=2, B=4, N=4)$ with 


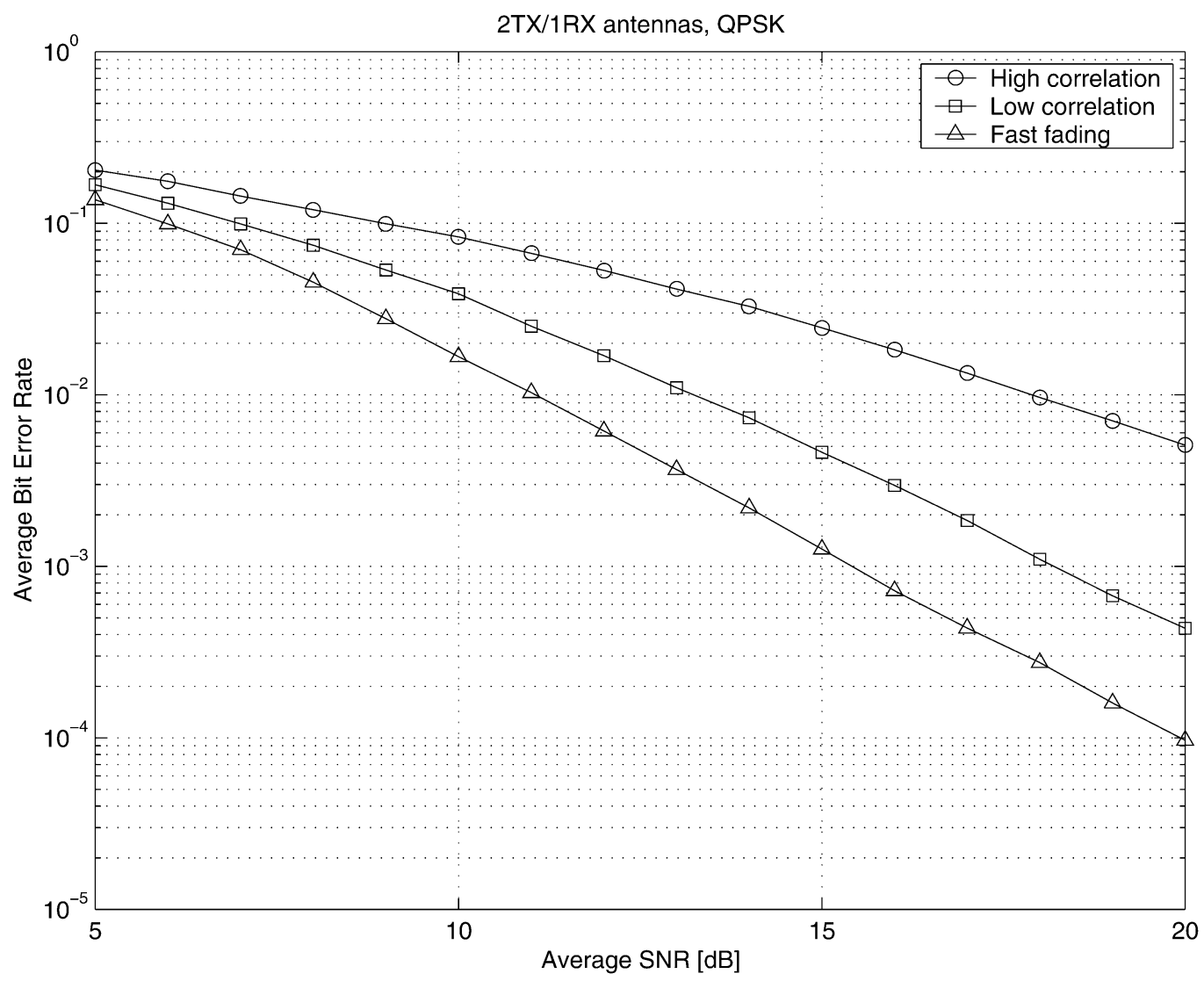

Fig. 3. ST code for two antennas, QPSK.

one receive antenna. The bit-error rate curves for the same code with two receive antennas are shown in Fig. 4. Both curves demonstrate that the spatio-temporal correlation has a significant impact on the performance. Moreover, it can be observed that in the low-correlation case, the bit-error probability curve becomes approximately parallel to the fast-fading bit-error probability curve at high SNR. Therefore, they achieve the same diversity level, validating our analysis.

Since we are not aware of any other code construction method for ST correlated channels, we compare our method with the ST codes of [4], [5] designed for fast-fading channels, and the ST code of [10] designed for quasi-static fading channels. These ST codes represent point solutions in the design space, and they are used to verify the performance of the codes obtained via the proposed systematic construction method in the special cases where previously known solutions exist.

Fig. 5 shows the performance of the ST codes constructed for a two-transmit-antenna system and QPSK modulation $(K=2, B=4$, $N=4$ ) with one receive antenna. All of these codes satisfy the uniqueness criterion. It is observed that all codes have essentially the same performance, with the ST code from [10] being a little better in the high-correlation case and being a little worse in the fast-fading case. Note that our systematic design method resulted in an ST code that achieves the same performance as the ST code of [5], which was found by computer search. The theoretical performance of the above codes was also compared. Table II shows the minimum norm product values $\delta_{\tau}$ corresponding to the $\tau$-length error events. The entries in the second row of the table show the $\delta_{\tau}$ values for the ST codes of [4], [5] and our method, while the third row contains the $\delta_{\tau}$ values for the ST code of [10]. The table entries were obtained by performing computer search. These $\delta_{\tau}$ values predict similar performance of the methods described in [4], [5], and the proposed approach, along with the slightly worse performance of the ST code of [10] at high SNR.
TABLE II

Minimum NoRm PRODUCTS FOR TwO ANTENNAS, QPSK

\begin{tabular}{|c||c|c|c|c|c|}
\hline$\tau$ & 2 & 3 & 4 & 5 & 6 \\
\hline$\delta_{\tau}$ & 24 & 32 & 64 & 128 & 256 \\
\hline$\delta_{\tau}[10]$ & 16 & 32 & 96 & 128 & 256 \\
\hline
\end{tabular}

TABLE III

Minimum Norm Products FOR THREE ANTENNAS, QPSK

\begin{tabular}{|c||c|c|c|c|}
\hline$\tau$ & 3 & 4 & 5 & 6 \\
\hline$\delta_{\tau}$ & 384 & 384 & 2304 & 6144 \\
\hline$\delta_{\tau}[10]$ & 216 & 576 & 1728 & 2880 \\
\hline
\end{tabular}

The bit-error rate curves for three transmit antennas and QPSK modulation ( $K=3, B=4, N=16$ ) with one receive antenna are depicted in Fig. 6. Our code (the ST code shown in Fig. 1) is compared to the ST code described in [10]. These codes also satisfy the uniqueness criterion. The performance of the two codes is almost identical, and the bit-error curves for the fast fading channel model and for the low correlation channel model are approximately parallel at high SNR. The ST code of [10], also found by computer search, performs slightly better in the high correlation case, which is expected since this code was designed for quasi-static channels. The minimum norm product values for the proposed method are given in the second row of Table III, and the third row contains the values for the ST code given in [10]. The theoretical norm product values confirm the tendencies observed in Fig. 6 .

The performance of our ST code constructed for three transmit antennas and 8PSK modulation ( $K=3, B=8, N=64)$ with two receive antennas is shown in Fig. 7. The bit-error rate curves of our four-antenna 4ASK ST code $(K=4, B=4, N=64)$ with one 


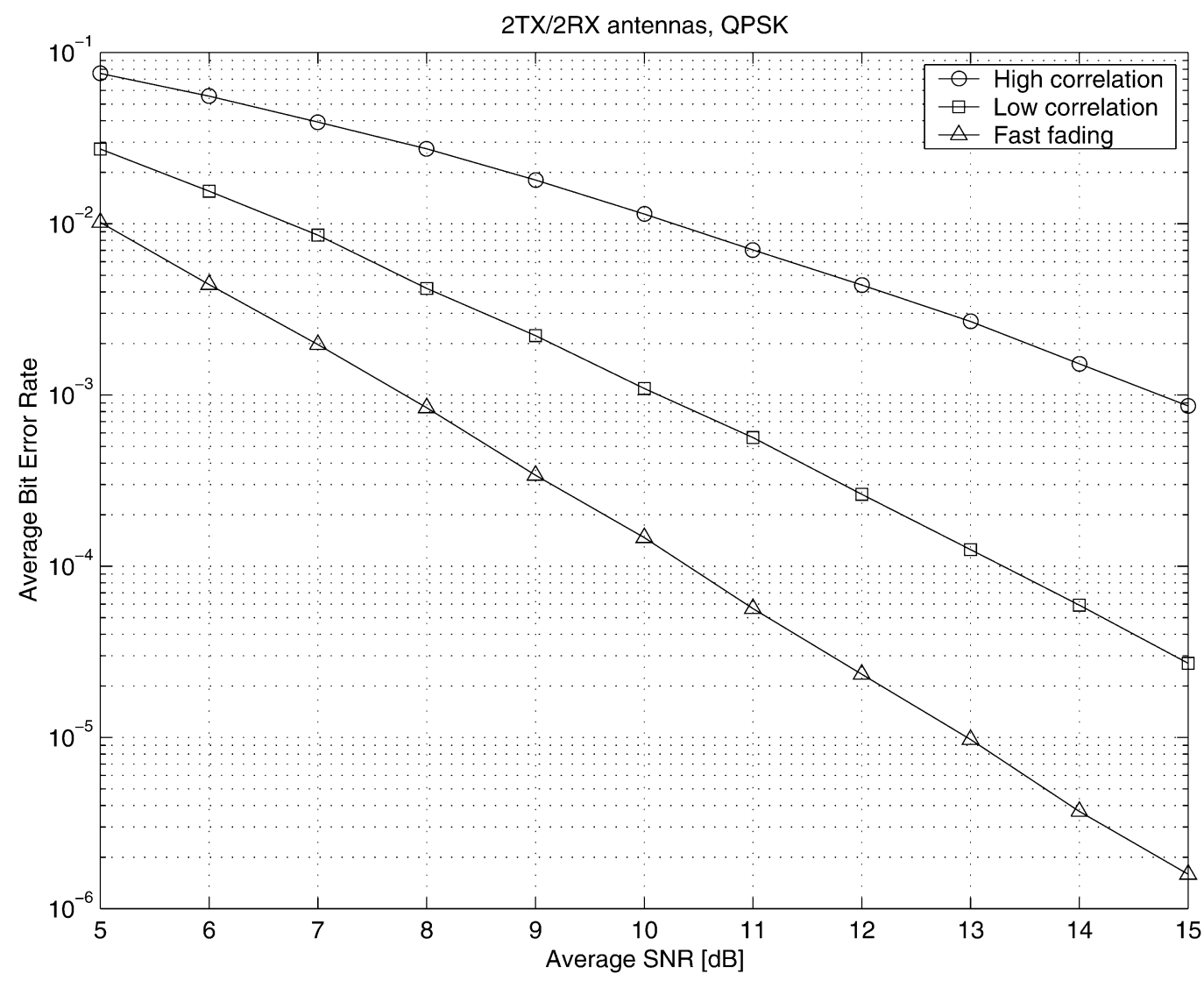

Fig. 4. ST code for two antennas, QPSK.

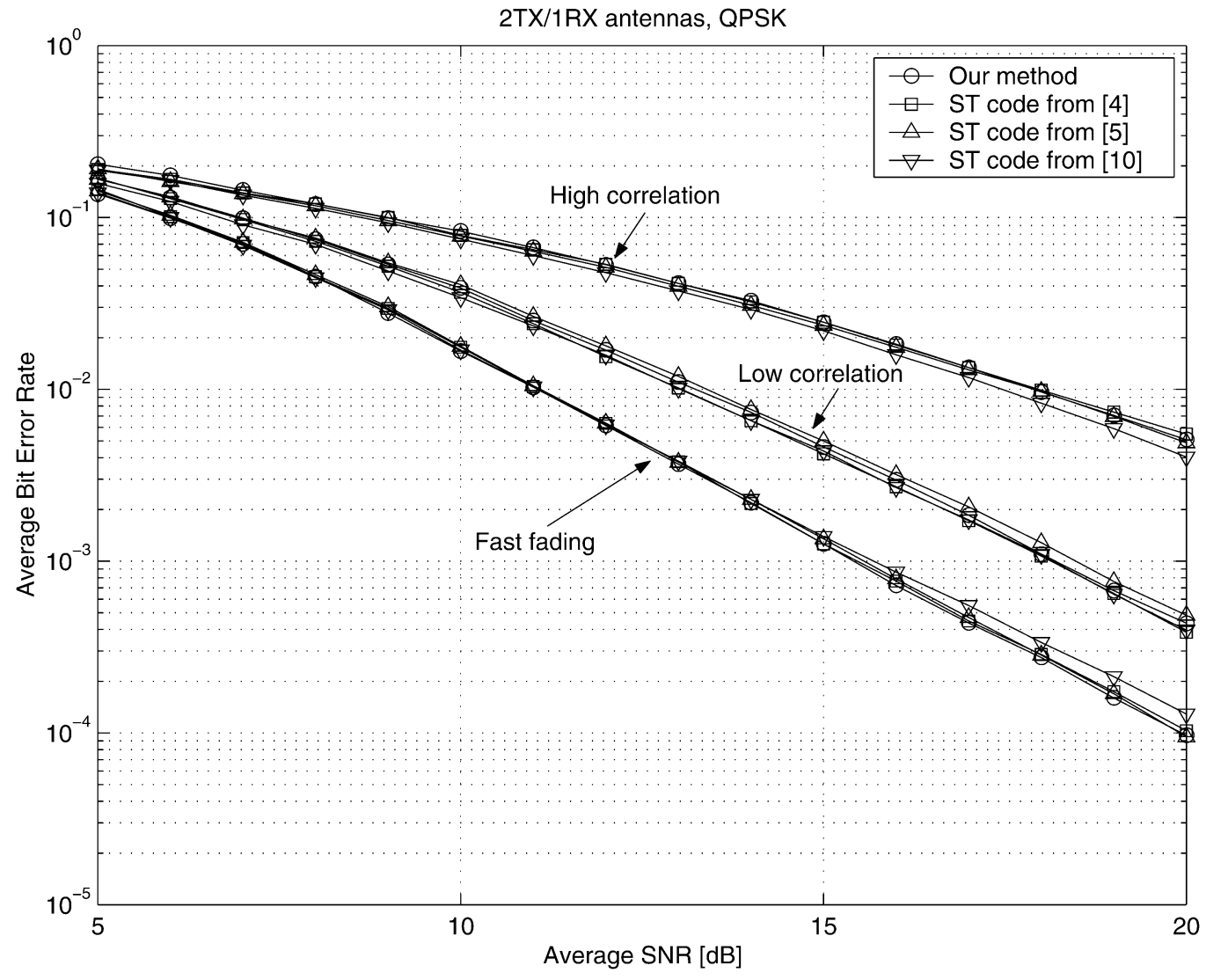

Fig. 5. ST codes for two antennas, QPSK. 


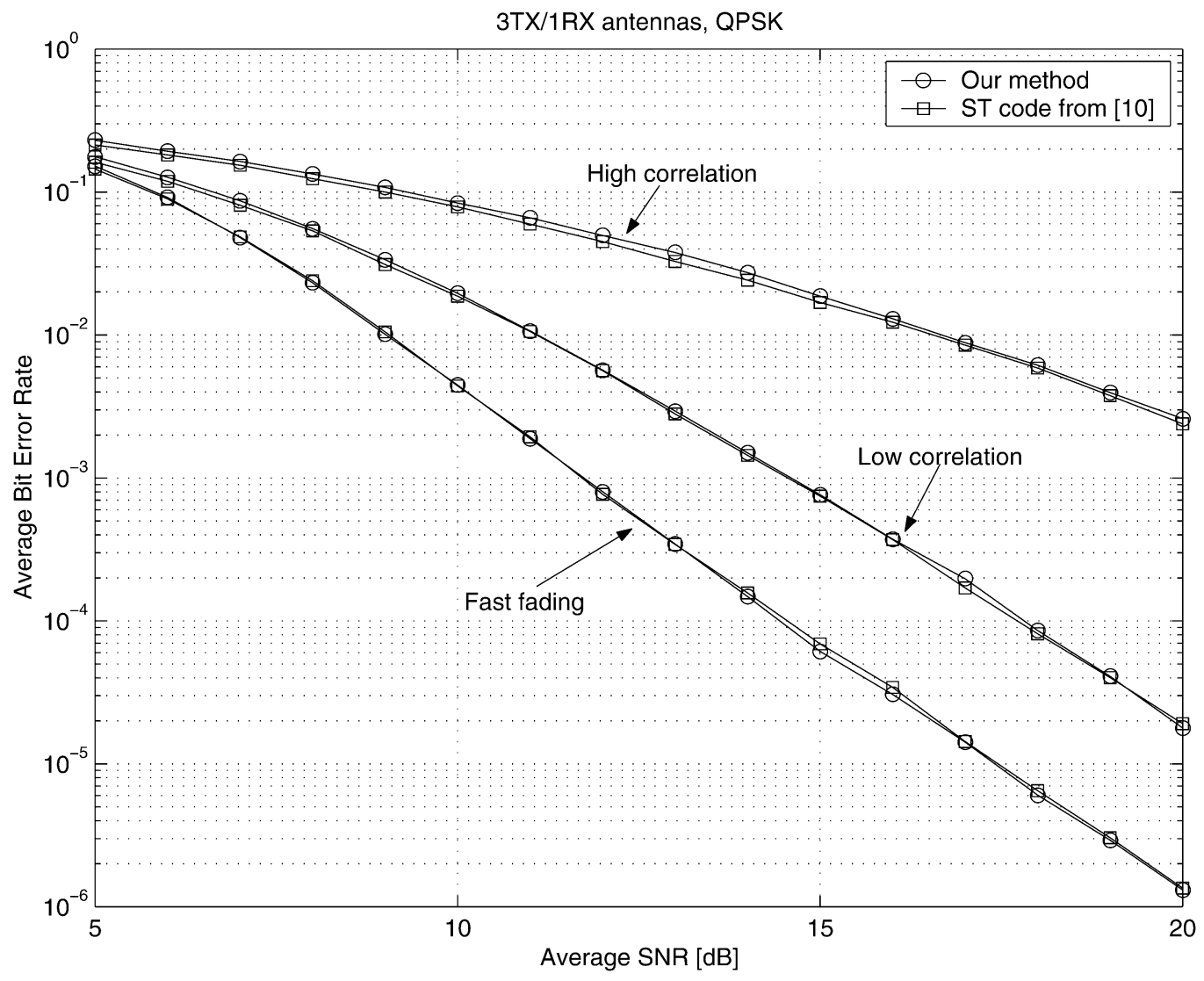

Fig. 6. ST codes for three antennas, QPSK.

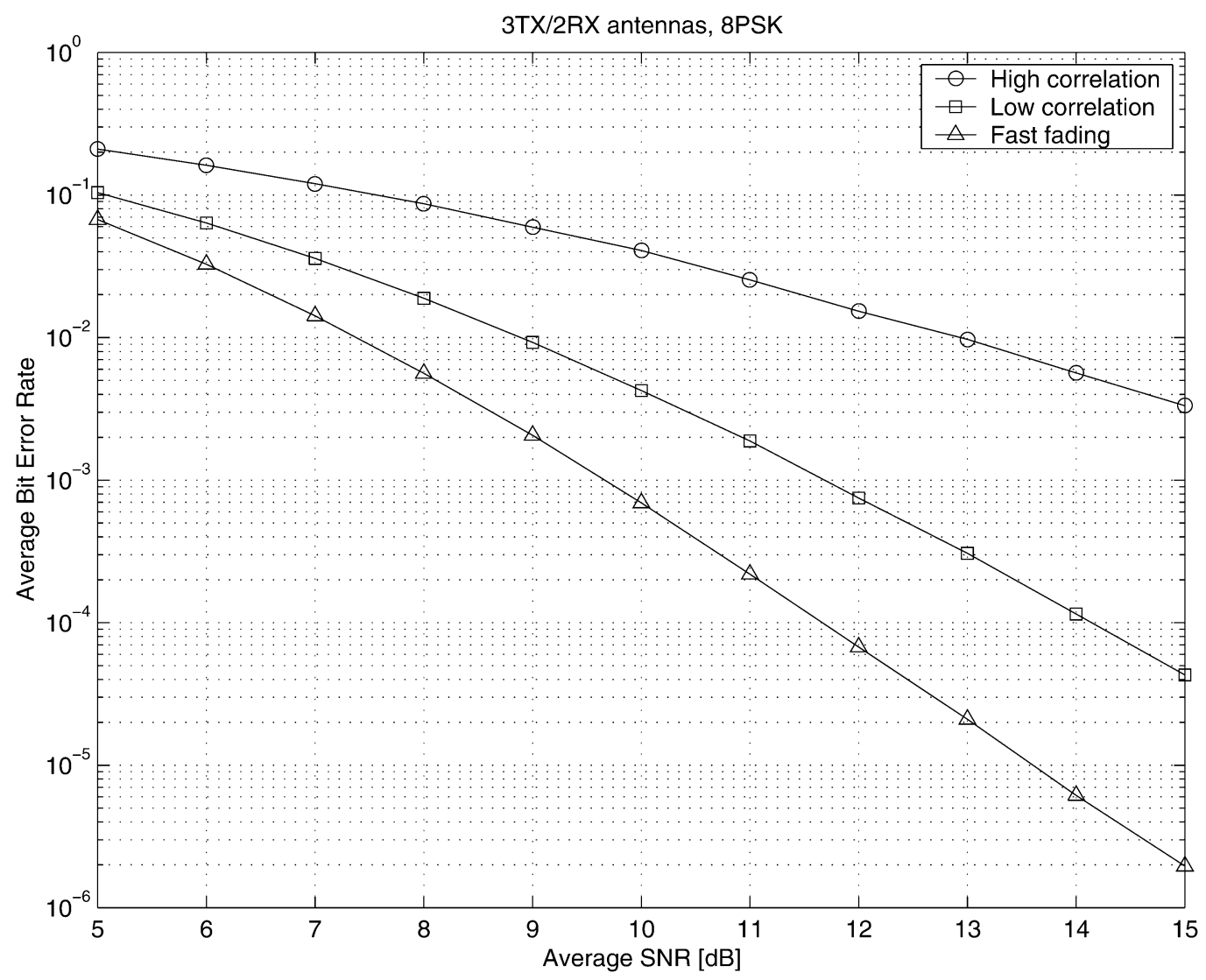

Fig. 7. ST codes for three antennas, 8PSK. 


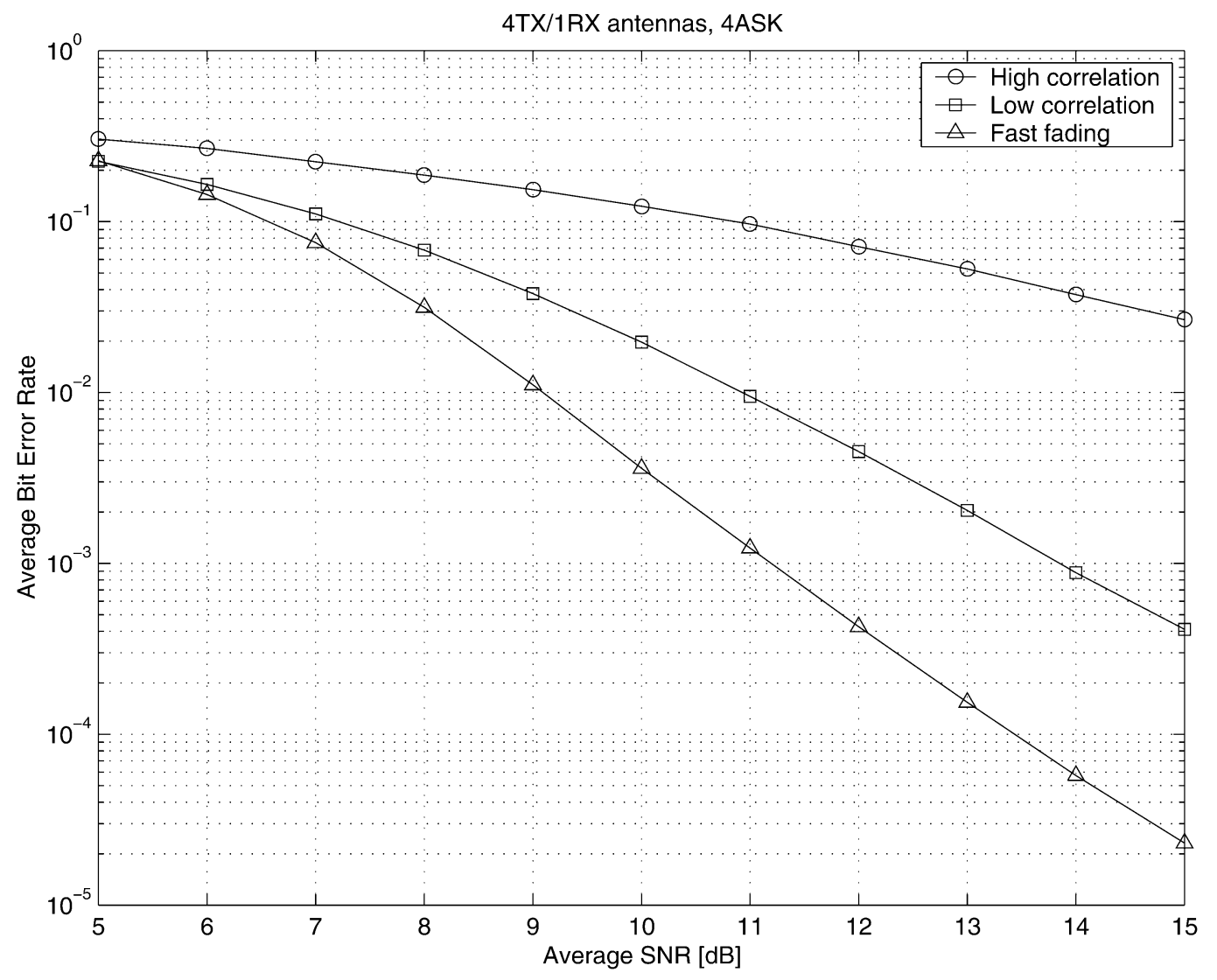

Fig. 8. ST codes for four antennas, 4ASK.

receive antenna can be observed in Fig. 8. The behavior of the curves are similar to that of the two- and three-transmit-antenna cases. To our knowledge, no ST codes have been published that we could compare these codes against.

\section{CONCLUSION}

We derived the performance criteria for ST correlated flat Rayleighfading channels with full-rank correlation matrix, and we developed a systematic ST trellis code design method for an arbitrary number of transmit antennas and any memoryless modulation. Based on the theoretical and experimental results, we can draw the following conclusion.

If the ST channel is not heavily correlated (i.e., the ST correlation matrix is of full rank), the ST code design problem for correlated channels can be reduced to the code design problem for fast fading channels, and it is possible to achieve the diversity level available in fast fading environment. Moreover, for communication systems having $K$ transmit and $L$ receive antennas, the ST-correlated channel model does not limit the maximum achievable diversity level: by increasing the number of encoder and decoder states (increasing the length of the shortest error event), arbitrarily high diversity order can be achieved.

The ST trellis codes constructed by the proposed method were also compared to existing ST codes that represent point solutions in our design space. The simulations showed that our systematic design procedure results in codes that have the same performance as the codes previously found by computer search, so our codes are expected to perform very well in cases where exhaustive computer search is not feasible. We also constructed ST trellis codes for three and four transmit antennas and 8PSK and 4ASK modulations. For these design parameters and non-quasi-static channel models, no other ST trellis codes exist in the literature.

\section{APPENDIX A}

For simplicity, we assume that $\boldsymbol{R}$ is $p$ by $p, \boldsymbol{R}^{\prime}$ is $m$ by $m$, and $\boldsymbol{\Delta}^{\prime}$ is $n$ by $m$, with $p \geq m \geq n$. In our case, $p=K L T, m=K L \tau$, and $n=L \tau$. Let us denote the positive and real eigenvalues of $\boldsymbol{R}$ by $\lambda_{1} \geq \lambda_{2} \geq \cdots \geq \lambda_{p}$. Using the singular value decomposition, $\boldsymbol{\Delta}^{\prime}$ can be expressed as $\boldsymbol{\Delta}^{\prime}=\boldsymbol{X}[\boldsymbol{\Sigma} 0] \boldsymbol{Y}^{H}$, where $\boldsymbol{X}$ is an $n$ by $n$ unitary matrix, $\boldsymbol{Y}$ is an $m$ by $m$ unitary matrix, $\boldsymbol{\Sigma}$ is an $n$ by $n$ diagonal matrix with the singular values along the diagonal, and $\mathbf{0}$ is an $n$ by $(m-n)$ zero matrix. The matrix $\boldsymbol{R}^{\prime}$ admits the spectral decomposition $\boldsymbol{R}^{\prime}=$ $\boldsymbol{U} \boldsymbol{\Lambda}^{\prime} \boldsymbol{U}^{H}$, with an $m$ by $m$ unitary matrix $\boldsymbol{U}$, and a diagonal matrix $\Lambda^{\prime}=\operatorname{diag}\left(\lambda_{1}^{\prime}, \lambda_{2}^{\prime}, \ldots, \lambda_{m}^{\prime}\right)$. The quantities $\lambda_{1}^{\prime} \geq \lambda_{2}^{\prime} \geq \cdots \geq \lambda_{m}^{\prime}$ are the real eigenvalues of $\boldsymbol{R}^{\prime}$. We can define $\boldsymbol{Z}$, the $m$ by $m$ unitary matrix, as $\boldsymbol{Z}=\boldsymbol{Y}^{H} \boldsymbol{U}$ and partition $\boldsymbol{Z}$ into an $n$ by $m$ matrix $\boldsymbol{Z}_{1}$, and an $(m-n)$ by $m$ matrix $\boldsymbol{Z}_{2}$ as

$$
\boldsymbol{Z}=\left[\begin{array}{l}
Z_{1} \\
Z_{2}
\end{array}\right] .
$$

The matrix $\boldsymbol{Q}=\boldsymbol{Z} \mathbf{\Lambda}^{\prime} \boldsymbol{Z}^{H}$ will have the same eigenvalues as $\boldsymbol{R}^{\prime}$. If $\boldsymbol{Q}$ is partitioned as

$$
\boldsymbol{Q}=\left[\begin{array}{ll}
\boldsymbol{Q}_{11} & \boldsymbol{Q}_{12} \\
\boldsymbol{Q}_{21} & \boldsymbol{Q}_{22}
\end{array}\right]
$$

where $\boldsymbol{Q}_{11}=\boldsymbol{Z}_{1} \boldsymbol{\Lambda}^{\prime} \boldsymbol{Z}_{1}^{H}$ is an $n$ by $n$ principal submatrix of $\boldsymbol{Q}$, $\Delta^{\prime} R^{\prime} \Delta^{\prime H}$ can be expressed as

$$
\boldsymbol{\Delta}^{\prime} \boldsymbol{R}^{\prime} \boldsymbol{\Delta}^{\prime H}=\boldsymbol{X} \boldsymbol{\Sigma} \boldsymbol{Q}_{11} \boldsymbol{\Sigma}^{H} \boldsymbol{X}^{H} .
$$

Since $\boldsymbol{\Delta}^{\prime}$ has full row rank, the matrix $\boldsymbol{\Sigma}$ has full rank. Using Fisher's inequality [14], it can be easily verified that $\boldsymbol{Q}_{11}$ also has full rank. 
Moreover, all matrices on the right-hand side of (26) are $n$ by $n$. As a consequence, we have the relationship

$$
\begin{aligned}
\operatorname{det}\left(\boldsymbol{\Delta}^{\prime} \boldsymbol{R}^{\prime} \boldsymbol{\Delta}^{\prime H}\right) & =\operatorname{det}\left(\boldsymbol{Q}_{11}\right) \operatorname{det}\left(\boldsymbol{\Sigma} \boldsymbol{\Sigma}^{H}\right) \operatorname{det}\left(\boldsymbol{X} \boldsymbol{X}^{H}\right) \\
& =\operatorname{det}\left(\boldsymbol{Q}_{11}\right) \operatorname{det}\left(\boldsymbol{\Delta}^{\prime} \boldsymbol{\Delta}^{\prime H}\right) .
\end{aligned}
$$

To obtain a lower bound on $\operatorname{det}\left(\boldsymbol{Q}_{11}\right)$, we use Cauchy's interlacing theorem [15] (also known as the inclusion principle [14]), stated as follows: Let $\boldsymbol{Q}$ be an $m$ by $m$ Hermitian matrix with real eigenvalues $\lambda_{1}^{\prime} \geq \lambda_{2}^{\prime} \geq \cdots \geq \lambda_{m}^{\prime}$. Furthermore, let $Q_{11}$ be an $n$ by $n(m \geq n)$ principal submatrix of $Q$, with real eigenvalues $\mu_{1} \geq \mu_{2} \geq \cdots \geq \mu_{n}$. Then we have $\lambda_{i}^{\prime} \geq \mu_{i} \geq \lambda_{m-n+i}^{\prime}$, for $i=1,2, \ldots, n$. Moreover, since $\boldsymbol{R}^{\prime}$ is a principal submatrix of $\boldsymbol{R}$, we can apply Cauchy's interlacing theorem again to obtain $\lambda_{i} \geq \lambda_{i}^{\prime} \geq \lambda_{p-m+i}$, for $i=1,2, \ldots, m$. Therefore, if we form the diagonal matrix $\Lambda_{\min }(n)$ from the $n$ smallest eigenvalues of $\boldsymbol{R}$ (i.e., $\left.\boldsymbol{\Lambda}_{\min }(n)=\operatorname{diag}\left(\lambda_{p-n+1}, \lambda_{p-n+2}, \ldots, \lambda_{p}\right)\right)$, we obtain the bound

$$
\operatorname{det}\left(\boldsymbol{Q}_{11}\right) \geq \operatorname{det}\left(\boldsymbol{\Lambda}_{\min }(n)\right) .
$$

Note that (28) also shows that $Q_{11}$ has full rank. Finally, combining (27) with (28) yields (4).

\section{APPENDIX B}

We will prove (21) by induction. In [12], closed-form expressions that relate the state sequence $\left\{S_{t}\right\}$ to the starting state $S_{0}$ and input source symbol sequence $\left\{b_{t}\right\}$ up to the length of the shortest error event were derived. In case of encoders having $N_{\min }$ states, the expression for $S_{K}$ becomes $S_{K}=\sum_{m=1}^{K-1} B^{K-1-m} b_{m}$, showing that the statement is true for $T=0$. Assume that the formula holds for $T-1$, i.e.,

$$
S_{T+K-1}=\sum_{m=T}^{T+K-2} B^{T+K-2-m} b_{m} .
$$

The state transition at time $t$ can also be expressed recursively as a function of the previous state $S_{t-1}$, and the previous source symbol $b_{t-1}[12]$ as

$$
S_{t}=B\left(S_{t-1} \bmod \left(B^{K-2}\right)\right)+b_{t-1} .
$$

Using (30) with $t=T+K$, and combining it with (29), we arrive at (21), proving that the formula also holds for $T$.

\section{REFERENCES}

[1] V. Tarokh, N. Seshadri, and A. Calderbank, "Space-time codes for high data rate wireless communication: Performance criterion and code construction," IEEE Transactions on Information Theory, vol. 44, pp. 744-765, Mar. 1998.

[2] J. Grimm, M. Fitz, and J. Krogmeier, "Further results on space-time coding for Rayleigh fading," in Proc. 36th Allerton Conf. Communications, Control and Computing, 1998, pp. 391-400.

[3] A. R. Hammons and H. El Gamal, "On the theory of space-time codes for PSK modulation," IEEE Trans. Inform. Theory, vol. 46, pp. 524-542, Mar. 2000.

[4] S. Zummo and S. Al-Semari, "Space-time coded QPSK for rapid fading channels," Proc. PIMRC, vol. 1, pp. 504-508, 2000.

[5] W. Firmanto, B. Vucetic, and J. Yuan, "Space-time TCM with improved performance on fast fading channels," IEEE Commun. Lett., vol. 5, pp. 154-156, Apr. 2001.

[6] H. Bolcskei and A. Paulraj, "Performance of space-time codes in the presence of spatial fading correlation," in Proc. Asilomar Conf. Signals, Systems and Computers, vol. 1, 2000, pp. 687-693.

[7] S. Siwamogsatham, M. Fitz, and J. Grimm, "A new view of performance analysis of transmit diversity schemes in correlated Rayleigh fading," IEEE Trans. Inform. Theory, vol. 48, pp. 950-956, Apr. 2002.
[8] S. Siwamogsatham and M. Fitz, "Robust space-time codes for correlated Rayleigh fading channels," in Proc. 38th Annual Allerton Conf. Communication, Control, and Computing, Oct. 2000. See Also IEEE Trans. Signal Processing, vol. 50, pp. 2408-2416, Oct. 2002.

[9] S. Alamouti, "A simple transmit diversity technique for wireless communications," IEEE J. Select. Areas Commun., vol. 16, pp. 1451-1458, Aug. 1998.

[10] Q. Yan and R. Blum, "Optimum space-time convolutional codes," Proc. WCNC, vol. 3, pp. 1351-1355, 2000.

[11] H. El Gamal, "On the robustness of space-time coding," IEEE Trans. Signal Processing, vol. 50, pp. 2417-2428, Oct. 2002.

[12] Z. Safar and K. J. R. Liu, "Systematic design of space-time trellis codes for diversity and coding advantages," EURASIP J. Appl. Signal Processing, Special Issue on Space-Time Coding and Its Applications, no. 3, pp. 221-235, Mar. 2002.

[13] , "Space-time correlation of MIMO flat Rayleigh fading channels," Proc. EUSIPCO, vol. 3, pp. 323-326, 2002.

[14] R. Horn and C. Johnson, Matrix Analysis. Cambridge, U.K.: Cambridge Univ. Press, 1985.

[15] G. Stewart and J. Sun, Matrix Perturbation Theory. New York: Academic, 1990

\section{On the Performance Analysis of Space-Time Codes in Quasi-Static Rayleigh-Fading Channels}

Myung-Kwang Byun, Daeyoung Park, Student Member, IEEE, and Byeong Gi Lee, Fellow, IEEE

\begin{abstract}
In this correspondence, we analyze the performance of space-time codes by deriving a new approximation of the frame error probabilities for space-time trellis-coded modulations over quasi-static Rayleigh-fading channels. We take advantage of two techniques, the modified bounding technique and the limiting-before-averaging technique, to tighten the upper bound. In addition, we establish a theorem that enables us to reduce the computation and memory needed to calculate the frame error rate (FER) approximation. The newly derived approximation is very tight, requires only the distance spectrum of the space-time code, and can be computed through single numerical integration. Numerical results exhibit that the new approximation is much closer to the simulation results than other existing bounds are, especially in the case of one receive antenna.
\end{abstract}

Index Terms-Distance spectrum, fading channels, frame error rate (FER), performance analysis, space-time codes.

\section{INTRODUCTION}

High data-rate transmission is strictly limited in wireless communications due to physical limitations, such as limited bandwidth, propagation loss, time variance, noise, interference, and multipath fading.

Manuscript received November 20, 2001; revised July 20, 2004. The material in this correspondence was presented in part at the IEEE International Conference on Communications, New York, NY, June 2002.

M.-K. Byun is with Samsung Electronics Co. Ltd., Suwon, Korea (e-mail: mk.byun@samsung.com).

D. Park was with the School of Electrical Engineering, Seoul National University, Seoul, 151-742, Korea. He is now with Samsung Advanced Institute of Technology, Kiheungeup, Yonginsi, Kyeongkido, Korea (e-mail: pdy@tsp.snu.ac.kr).

B. G. Lee is with the School of Electrical Engineering, Seoul National University, Seoul, 151-742, Korea (e-mail: blee@tsp.snu.ac.kr).

Communicated by G. Caire, Associate Editor for Communications.

Digital Object Identifier 10.1109/TIT.2004.836871 